\title{
Chemical Moiety
}

National Cancer Institute

\section{Source}

National Cancer Institute. Chemical Moiety. NCI Thesaurus. Code C103246.

A single entity that can be represented as a set of atoms usually connected through a continuous set of covalent bonds. 